\title{
MODIFIED DIAGONALS AND LINEAR RELATIONS BETWEEN SMALL DIAGONALS
}

\author{
HUNTER SPINK
}

\begin{abstract}
We prove that the vanishings of the modified diagonal cycles of Gross and Schoen govern the $\mathbb{Z}$-linear relations between small $m$-diagonals $\mathrm{pt}^{\{1, \ldots, n\} \backslash A} \times \Delta_{A}$ in the rational Chow ring of $X^{n}$ for $A$ ranging over $m$ element subsets of $\{1, \ldots, n\}$. Our results generalize to arbitrary symmetric classes in place of the diagonal in $X^{m}$, and with different types of inclusions $A^{\bullet}\left(X^{m}\right)_{\mathbb{Q}}^{S_{m}} \hookrightarrow A^{\bullet}\left(X^{n}\right)_{\mathbb{Q}}$.

The combinatorial heart of this paper, which may be of independent interest, is showing the $\mathbb{Z}$-linear relations between elementary symmetric polynomials $e_{k}\left(x_{a_{1}}, \ldots, x_{a_{m}}\right) \in \mathbb{Z}\left[x_{1}, \ldots, x_{n}\right]$ are generated by the $S_{n}$-translates of a certain alternating sum over the facets of a hyperoctahedron.
\end{abstract}

\section{INTRODUCTION}

Let $X$ be a smooth projective variety, and denote the rational Chow ring of a variety $Y$ by

$$
A^{\bullet}(Y)_{\mathbb{Q}}:=A^{\bullet}(Y) \otimes \mathbb{Q} .
$$

Fix an integer $m \geq 0$ and a symmetric class

$$
\alpha \in A^{\bullet}\left(X^{m}\right)_{\mathbb{Q}}^{S_{m}} \subset A^{\bullet}\left(X^{m}\right)_{\mathbb{Q}} .
$$

A typical example of such an $\alpha$ would be the class of the diagonal $\Delta_{m} \subset X^{m}$. We denote by $\Delta_{A}$ the diagonal in $X^{A}$ for any set $A$.

Given a subset $A \subset\{1, \ldots, n\}$ of size $m$, we have the following inclusions $A^{\bullet}\left(X^{m}\right)_{\mathbb{Q}}^{S_{m}}=A^{\bullet}\left(X^{A}\right)_{\mathbb{Q}}^{S_{m}} \hookrightarrow A^{\bullet}\left(X^{n}\right)_{\mathbb{Q}}$ :

- pullback along a projection $X^{n} \rightarrow X^{A}$, and

- pushforward along an inclusion $X^{A} \cong\{\mathrm{pt}\}^{\{1, \ldots, n\} \backslash A} \times X^{A} \hookrightarrow X^{n}$.

These are representative of the types of inclusions we will consider, and after choosing such a system of inclusions we denote by

$$
\alpha(A) \in A^{\bullet}\left(X^{n}\right)_{\mathbb{Q}}
$$

the image of $\alpha$ under such a map associated to an $m$-element set $A \subset\{1, \ldots, n\}$.

In this paper we classify the $\mathbb{Z}$-linear relations that can occur between such $\alpha(A)$, i.e. we classify kernels of maps $\mathbb{Z}^{\left(\begin{array}{l}n \\ m\end{array}\right)} \rightarrow A^{\bullet}\left(X^{n}\right)_{\mathbb{Q}}$ given by $A \mapsto \alpha(A)$. Surprisingly, there are very few possibilities, each of which is a group generated by the $S_{n}$-translates of a single relation which we call for reasons that will soon become apparent a "hyperoctahedral relation".

A very special case is the main application of this paper. In GS95, Gross and Schoen defined a certain "modified diagonal cycle" on $X^{k}$, given by

$$
\Delta_{k}^{\prime}:=\sum_{\emptyset \neq B \subset\{1, \ldots, k\}}(-1)^{k-|B|} \Delta_{B}(B) \in A^{\bullet}\left(X^{k}\right)_{\mathbb{Q}}
$$


where $\Delta_{B}(B)$ is defined to be the pushforward of $\Delta_{B}$ under $X^{B} \cong\{\mathrm{pt}\}\{1, \ldots, k\} \backslash B \times$ $X^{B} \hookrightarrow X^{k}$. In general, the class depends on the choice of pt $\in X$, and the vanishing has been intensely studied in the context of diagonal decompositions in Chow groups. In particular, we have the following incomplete list of results.

- In GS95, Gross and Schoen showed that

$-\Delta_{k}^{\prime}=0$ precisely if $k \geq 2$ for $X=\mathbb{P}^{1}$,

$-\Delta_{k}^{\prime}=0$ precisely if $k \geq 3$ for $X$ of genus 1 , and

$-\Delta_{3}^{\prime}=0$ for $X$ a hyperelliptic curve with pt $\in X$ a Weierstrass point.

- In [BV04, Beauville and Voisin showed that

$-\Delta_{3}^{\prime}=0$ on a $K 3$-surface $X$ if pt $\in X$ lies on a rational curve.

- In O'G14, O'Grady showed that

- If $\Delta_{m}^{\prime}=0$ then $\Delta_{m+s}^{\prime}=0$ for all $s \geq 0$ for $X$ any smooth projective variety.

- In Voi15, Voisin showed that

- if $X$ is a smooth projective connected variety of dimension $n$ swept out by irreducible curves of genus g supporting a zero-cycle rationally equivalent to pt $\in X$, then we have $\Delta_{m}^{\prime}=0$ for $m \geq(n+1)(g+1)$.

- In [MY16], Moonen and Yin showed that

$-\Delta_{n}^{\prime}=0$ on a $g$-dimensional abelian variety precisely when $n \geq 2 g+1$, and

$-\Delta_{n}^{\prime}=0$ on a curve of genus $g$ whenever $n \geq g+2$ (which is sharp for a generic pointed curve, see Qiz14).

We will show that the minimal $k \leq m$ such that $\Delta_{k}^{\prime}=0$ determines the $\mathbb{Z}$-linear relations between the $\left(\begin{array}{c}n \\ m\end{array}\right)$ classes $\Delta_{A}(A) \in A^{\bullet}\left(X^{n}\right)_{\mathbb{Q}}$ for $A \subset\{1, \ldots, n\}$ ranging over $m$-element subsets, which will be generated by the $S_{n}$-translates of a single " $k$-hyperoctahedral $m$-relation". As it is known (and easy to show) that only for $X=$ pt or $\mathbb{P}^{1}$ do we have $\Delta_{2}^{\prime}=0$, we may deduce as a formal consequence the set of such $\mathbb{Z}$-linear relations for all pairs $m \leq n$ for

- $X$ of genus $\leq 1$,

- $X$ a $g$-dimensional abelian variety,

- $X$ a generic pointed curve of genus $g$,

- $X$ a $K_{3}$ surface and pt $\in X$ lying on a rational curve, and

- $X$ hyperelliptic with pt $\in X$ a Weierstrass point.

Moreover, if $\Delta_{k}^{\prime}=0$ then the $k$-hyperoctahedral $m$-relations are a subset of the relations satisfied by the $\Delta_{A}(A)$ classes (which may turn out to be all such relations if $k$ happens to be minimal).

More generally, for an arbitrary symmetric class $\alpha \in A^{\bullet}\left(X^{m}\right)_{\mathbb{Q}}^{S_{m}}$, if we define $\alpha_{B}$ to be the pushforward of $\alpha$ along the projection $X^{m} \rightarrow X^{B}$ and $\alpha_{B}(B)$ analogously to $\Delta_{B}(B)$, then the vanishings of

$$
\alpha_{k}^{\prime}:=\sum_{B \subset\{1, \ldots, k\}}(-1)^{k-|B|} \alpha_{B}(B) \in A^{\bullet}\left(X^{k}\right)_{\mathbb{Q}}^{S_{k}}
$$

similarly control the $\mathbb{Z}$-linear relations between $\alpha(A)$ classes in $A^{\bullet}\left(X^{n}\right)_{\mathbb{Q}}$, which again arise as "hyperoctahedral $m$-relations" (note that $\Delta_{\emptyset}=0$ but $\alpha_{\emptyset}$ need not be zero).

As it turns out, the "hyperoctahedral relations" govern the $\mathbb{Z}$-linear relations between elementary symmetric polynomials $e_{k}\left(x_{a_{1}}, \ldots, x_{a_{m}}\right) \in \mathbb{Z}\left[x_{1}, \ldots, x_{n}\right]$ where $\left\{a_{1}, \ldots, a_{m}\right\} \subset\{1, \ldots, n\}$ ranges over $m$-element subsets, and the study of these 
relations between elementary symmetric polynomials forms the combinatorial heart of this paper.

The geometric heart of this paper is a Chow motive computation, which allows us to extract useful information about classes in $A^{\bullet}\left(X^{m}\right)_{\mathbb{Q}}$ despite having essentially no information about the ring itself. We will decompose the diagonal class in $A^{\bullet}\left(\left(X^{m}\right)^{2}\right)_{\mathbb{Q}}$ in such a way that convolving with the pieces yields a system of orthogonal idempotent endomorphisms of $A^{\bullet}\left(X^{m}\right)_{\mathbb{Q}}$, which consequently decomposes $A^{\bullet}\left(X^{m}\right)_{\mathbb{Q}}$ into the direct sum of the images of the idempotents. Our key insight is that we can produce such a decomposition where the non-zero components of $\alpha$ in these summands govern the $\mathbb{Z}$-linear relations between the $\alpha(A)$ classes.

The structure of this paper is as follows.

- In Section 2 we describe the "hyperoctahedral relations" and state our main results.

- In Section 3 we prove our main combinatorial result Theorem 2.3 classifying the $\mathbb{Z}$-linear relations between polynomials $e_{k}\left(x_{a_{1}}, \ldots, x_{a_{m}}\right) \in \mathbb{Z}\left[x_{1}, \ldots, x_{n}\right]$ where $\left\{a_{1}, \ldots, a_{m}\right\} \subset\{1, \ldots, n\}$ ranges over $m$ element subsets.

- In Section 4 we prove our main geometric result Theorem 2.5 which specializes to the modified diagonal result mentioned previously.

\section{Hyperoctahedral RELATIONS AND STATEMENT OF RESUlts}

To state our results, it will be useful to notate the $\left(\begin{array}{l}n \\ m\end{array}\right)$ natural inclusions

$$
\mathbb{Z}\left[x_{1}, \ldots, x_{m}\right]^{S_{m}} \hookrightarrow \mathbb{Z}\left[x_{1}, \ldots, x_{n}\right]
$$

indexed by subsets $A \subset\{1, \ldots, n\}$ of size $m$.

Definition 2.1. Denote by $e_{k}\left(x_{1}, \ldots, x_{m}\right) \in \mathbb{Z}\left[x_{1}, \ldots, x_{m}\right]$ the $k$ 'th elementary symmetric polynomial. For a symmetric polynomial $f \in \mathbb{Z}\left[x_{1}, \ldots, x_{m}\right]^{S_{m}}$ and $A=$ $\left\{a_{1}, \ldots, a_{m}\right\} \subset\{1, \ldots, n\}$ an $m$-element set, we denote

$$
f(A):=f\left(x_{a_{1}}, \ldots, x_{a_{m}}\right) \in \mathbb{Z}\left[x_{1}, \ldots, x_{n}\right] .
$$

The " $k$-hyperoctahedral $m$-relations" which we will shortly define are $\mathbb{Z}$-linear relations which generate all $\mathbb{Z}$-linear relations between the polynomials $e_{k-1}(A)$ in $\mathbb{Z}\left[x_{1}, \ldots, x_{n}\right]$ for fixed $k$ and $A$ ranging over $m$-element subsets of $\{1, \ldots, n\}$. They are the $S_{n}$-translates of a single relation in $\mathbb{Z}\left[x_{1}, \ldots, x_{m+k}\right]$ between the $e_{k-1}(A)$ 's with $A \subset\{1, \ldots, m+k\}$ ranging over subsets of size $m$.

As an example, suppose we let $m=3$ and $k=3$. Then we can view

$$
\sum_{|A|=3} \lambda_{A} e_{2}(A)=0
$$

as describing a formal $\mathbb{Z}$-linear combination of triangles $\{i, j, k\} \subset\{1, \ldots, n\}$ such that after replacing each $\{i, j, k\}$ with the sum of its three edges $\{i, j\}+\{j, k\}+\{i, k\}$, the sum becomes zero.

If we have a triangulation of a surface such that the face map has chromatic number two, then we can alternately sum the triangles on the surface to get such a relation. The smallest non-trivial instance of this occurs for an octahedron. The sum of $\{i, j, k\}$ over all dark triangles minus the sum over light triangles in the octahedron 


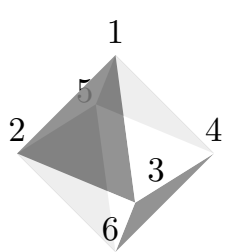

is what we will call a 3 -hyperoctahedral 3-sum, yielding the relation

$$
\begin{aligned}
& e_{2}(\{1,2,3\})+e_{2}(\{1,4,5\})+e_{2}(\{6,3,4\})+e_{2}(\{6,2,5\}) \\
= & e_{2}(\{1,3,4\})+e_{2}(\{1,2,5\})+e_{2}(\{6,2,3\})+e_{2}(\{6,4,5\}) .
\end{aligned}
$$

We will in fact show that such relations generate all $\mathbb{Z}$-linear relations between the $e_{2}(A)$ with $|A|=3$. As we will see later, the $\mathbb{Z}$-linear relations between the polynomials $e_{m-1}(A)$ for $|A|=m$ correspond to an alternating sum over the facets of an $m$-dimensional hyperoctahedron, which we will call an $m$-hyperoctahedral $m$-sum.

Definition 2.2. Given a set $B \subset\{1, \ldots, n\}$ of size $m-k$ and disjoint sets $C_{i}=$ $\left\{c_{i, 0}, c_{i, 1}\right\} \subset\{1, \ldots, n\} \backslash B$ for $i=1, \ldots, k$, we say that the element

$$
\sum_{\left(\epsilon_{1}, \ldots, \epsilon_{k}\right) \in\{0,1\}^{k}}(-1)^{\sum_{i=1}^{k} \epsilon_{i}} B \sqcup\left\{c_{1, \epsilon_{1}}, \ldots, c_{k, \epsilon_{k}}\right\}
$$

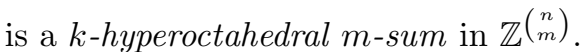

Define $G_{k} \subset \mathbb{Z}^{\left(\begin{array}{c}n \\ m\end{array}\right)}$ ) to be the subgroup generated by $k$-hyperoctahedral $m$-sums (we supress the dependence on $m$ and $n$ in the notation).

The parameter $k$ may be thought of as the "dimension" of the hyperoctahedron, and when $k<m$ this expression can be thought of as an (iterated) cone with apex(es) $B$ over a $k$-hyperoctahedral $k$-sum.

The reason for introducing this extra parameter $k$ lies in the following theorem, which is our central combinatorial result.

Theorem 2.3. Let $k \leq m \leq n$ be integers. Then the kernel of the map $\mathbb{Z}^{\left(\begin{array}{l}n \\ m\end{array}\right)} \rightarrow$ $\mathbb{Z}\left[x_{1}, \ldots, x_{n}\right]$ given by $A \mapsto e_{k}(A)$ is $G_{k+1}$, i.e. the $\mathbb{Z}$-linear relations between the $e_{k}(A)$ 's are generated by the $(k+1)$-hyperoctahedral $m$-sums.

Furthermore, we have the sequence of inclusions

$$
0=G_{m+1} \subset G_{m} \subset \ldots \subset G_{0}=\mathbb{Z}^{\left(\begin{array}{c}
n \\
m
\end{array}\right)} .
$$

Corollary 2.4. If $f \in \mathbb{Z}\left[x_{1}, \ldots, x_{m}\right]^{S_{m}}$ is a symmetric polynomial, then the kernel of the map $\mathbb{Z}^{\left(\begin{array}{c}n \\ m\end{array}\right)} \rightarrow \mathbb{Z}\left[x_{1}, \ldots, x_{n}\right]$ given by $A \mapsto f(A)$ is $G_{k+1}$, where $k$ is the largest number of distinct $x_{i}$ to appear in a non-zero monomial.

Proof of Corollary 2.4. For $B=\left(b_{1}, \ldots, b_{j}\right)$ with $b_{1} \leq b_{2} \leq \ldots \leq b_{j}$, if we let $g_{B}$ be the sum of all distinct monomials $x_{i_{1}}^{b_{1}} \cdots x_{i_{j}}^{b_{j}}$, then we can write $f=\sum_{B} \lambda_{B} g_{B}$ for some coefficients $\lambda_{B}$, and the $\mathbb{Z}$-linear relations between $f(A)$ are the intersections of the relation groups for each $g_{B}$ such that $\lambda_{B} \neq 0$. But it is easy to see that the relations between $g_{B}(A)$ are identical to the relations between $e_{|B|}(A)$, which by Theorem 2.3 is given by $G_{|B|+1}$. As the $G$ 's are nested, the result follows.

We are now in a position to state our geometric results properly. In a product space, we use $\nabla$ to denote intersection product of pullbacks from disjoint factors. 
Theorem 2.5. Let $X$ be a smooth projective variety and let $\gamma \in A^{\bullet}(X)_{\mathbb{Q}}$ be a class for which there exists a $\gamma^{\prime}$ with $\int_{X \rightarrow \mathrm{pt}} \gamma \cup \gamma^{*}=1$. Then given $\alpha \in A^{\bullet}\left(X^{m}\right)_{\mathbb{Q}}^{S_{m}}$,

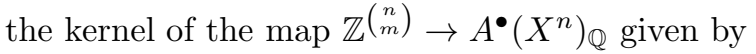

$$
A \mapsto \alpha(A):=\gamma^{\bigotimes\{1, \ldots, n\} \backslash A} \otimes \alpha
$$

is of the form $G_{k+1}$ for some $-1 \leq k \leq m$. If $\alpha=0$ then $k=-1$, otherwise $k$ is the largest number $\leq m$ such that

$$
0 \neq \alpha_{k}^{\prime}:=\sum_{B \subset\{1, \ldots, k\}}(-1)^{k-|B|} \alpha_{B}(B) \in A^{\bullet}\left(X^{k}\right)_{\mathbb{Q}}
$$

where we define $\alpha_{B} \in A^{\bullet}\left(X^{B}\right)_{\mathbb{Q}}^{S_{B}}$ to be the projection of $\left(\gamma^{*}\right)^{\bigotimes\{1, \ldots, m\} \backslash B} \cup \alpha$ to $X^{B}$ and $\alpha_{B}(B)=\gamma^{\bigotimes\{1, \ldots, n\} \backslash B} \otimes \alpha_{B}$.

Corollary 2.6. Let $\Delta_{m}$ be the diagonal in $A^{\bullet}\left(X^{m}\right)_{\mathbb{Q}}$, and pt $\in X$. Then the kernel of the map $\mathbb{Z}^{\left(\begin{array}{c}n \\ m\end{array}\right)} \rightarrow A^{\bullet}\left(X^{n}\right)_{\mathbb{Q}}$ given by

$$
A \mapsto \Delta_{m}(A)=\mathrm{pt}^{\{1, \ldots, n\} \backslash A} \times \Delta_{A}
$$

is $G_{\ell}$ for $\ell \leq m$ the smallest number such that the modified diagonal

$$
\Delta_{\ell}^{\prime}:=\sum_{\emptyset \neq B \subset\{1, \ldots, \ell\}}(-1)^{\ell-|B|} \Delta_{B}(B) \in A^{\bullet}\left(X^{\ell}\right)_{\mathbb{Q}}
$$

vanishes, where $\Delta_{B}(B)=\mathrm{pt}^{\{1, \ldots, \ell\} \backslash B} \times \Delta_{B}$. If $\ell=0$ then there are no relations.

Proof. Take $\gamma=$ [pt]. By ['G14], if $\Delta_{k}^{\prime}$ vanishes then $\Delta_{k+s}^{\prime}$ vanishes for all $s \geq 0$, so $\ell=k+1$ where $k$ is the largest number such that $\Delta_{k}^{\prime}$ does not vanish.

\section{Proof of Theorem 2.3}

In this section, we prove our main combinatorial result Theorem 2.3

Proof of Theorem 2.3. First, we will show that there are no linear relations between $e_{k}(A)$ for $A$ ranging over $m$ element subsets of $\{1, \ldots, m+k\}$, and then we will show that using $(k+1)$-hyperoctahedral $m$-relations we can reduce every relation down to one where $A$ ranges over fixed $m$-element subsets of $\{1, \ldots, m+k\}$.

To show the linear independence, note there are $\left(\begin{array}{c}m+k \\ m\end{array}\right)$ polynomials of the form $e_{k}(A)$ with $A$ of size $m$ inside $\{1, \ldots, m+k\}$, and this is equal to $\left(\begin{array}{c}m+k \\ k\end{array}\right)$, the number of $k$-products of distinct monomials $x_{i}$ with $i \in\{1, \ldots, m+k\}$. Hence, to show the linear independence it suffices to show that we can write each of these monomials as a $\mathbb{Q}$-linear combination of the $e_{k}(A)$ 's with $A$ of size $m$ in $\{1, \ldots, m+k\}$.

We will do this by inductively showing that all monomials of the form $x_{i_{1}} \ldots x_{i_{\ell}} e_{k-\ell}(B)$ lie in the $\mathbb{Q}$-linear span of the $e_{k}(A)$ with $i_{1}, \ldots, i_{\ell} \in\{1, \ldots, m+k\}$ distinct and $B \subset\{1, \ldots, m+k\} \backslash\left\{i_{1}, \ldots, i_{\ell}\right\}$ a subset of size $m$. Indeed, this is true for $\ell=0$. Suppose the result is true for $\ell-1$, we will show it is true for $\ell$. Indeed,

$$
\begin{aligned}
(m-(k-\ell)) x_{i_{1}} \ldots x_{i_{\ell}} e_{k-\ell}(B)= & x_{1} \ldots x_{i_{\ell-1}} \sum_{b \in B} e_{k-\ell+1}\left(\left\{i_{\ell}\right\} \sqcup B \backslash\{b\}\right)- \\
& x_{1} \ldots x_{i_{\ell-1}}(m-(k-\ell+1)) e_{k-\ell+1}(B) .
\end{aligned}
$$

Taking $\ell=k$ now shows that each such monomial is a $\mathbb{Q}$-linear combination of the $e_{k}(A)$ 's as desired. 
Now, using the $(k+1)$-hyperoctahedral $m$-relations, we will show that every relation can be reduced down to one where the $A$ range over $m$-element subsets of $\{1, \ldots, m+k\}$. We proceed by induction on $k$. For $k=0$ the result is trivial, so now assume that $k>0$. We are done if $n \leq m+k$, so assume that $n>m+k$. Then by assumption we have a relation

$$
0=\sum \lambda_{A} e_{k}(A)=x_{n} \sum_{n \in A} \lambda_{A} e_{k-1}(A \backslash n)+\sum_{n \in A} \lambda_{A} e_{k}(A \backslash n)+\sum_{n \notin A} \lambda_{A} e_{k}(A) .
$$

By the induction hypothesis, we know that $\sum_{n \in A} \lambda_{A} e_{k-1}(A \backslash n)$ is the sum of $k$-hyperoctahedral $(m-1)$-relations, so for each $i$ there exists an $m$-element set $B^{i} \subset\{1, \ldots, n-1\}$ and disjoint pairs $C_{j}^{i}=\left\{c_{j, 0}^{i}, c_{j, 1}^{i}\right\} \subset\{1, \ldots, n-1\} \backslash B^{i}$ for $1 \leq j \leq k$ such that

$$
\sum_{n \in A} \lambda_{A}(A \backslash n)=\sum_{i} \sum_{\left(\epsilon_{1}, \ldots, \epsilon_{k}\right) \in\{0,1\}^{k}}(-1)^{\sum \epsilon_{i}} B^{i} \bigsqcup\left\{c_{1, \epsilon_{1}}^{i}, \ldots, c_{k, \epsilon_{k}}^{i}\right\} .
$$

As $n-1 \geq m+k$ and only $m+k-1$ elements are used in the $i$ 'th hyperoctahedral sum, there exists an element $r_{i} \in\{1, \ldots, n-1\}$ not used in the $i$ 'th sum. Then letting $c_{k+1,0}^{i}=n$ and $c_{k+1,1}^{i}=r_{i}$, we have

$$
\begin{aligned}
\sum_{n \in A} \lambda_{A} A= & \sum_{i} \sum_{\left(\epsilon_{1}, \ldots, \epsilon_{k+1}\right) \in\{0,1\}^{k+1}}(-1)^{\sum \epsilon_{i}} B^{i} \bigsqcup\left\{c_{1, \epsilon_{1}}^{i}, \ldots, c_{k+1, \epsilon_{k+1}}^{i}\right\} \\
& +\sum_{i} \sum_{\left(\epsilon_{1}, \ldots, \epsilon_{k}\right) \in\{0,1\}^{k}}(-1)^{\sum \epsilon_{i}}\left\{r_{i}\right\} \cup B^{i} \bigsqcup\left\{c_{1, \epsilon_{1}}^{i}, \ldots, c_{k, \epsilon_{k}}^{i}\right\},
\end{aligned}
$$

where the first term on the right hand side is a sum of $(k+1)$-hyperoctahedral $m$ sums, and the second term does not involve $n$. Hence using $(k+1)$-hyperoctahedral $m$-relations we can reduce to a situation where $A \subset\{1, \ldots, n-1\}$. Repeating this we eventually reduce down to $A \subset\{1, \ldots, m+k\}$, and the result follows.

Finally, the nesting of the $G_{i}$ follows as given a relation between $e_{k}(A)$ 's, applying the operator $\frac{1}{m-k+1} \sum_{i=1}^{n} \frac{\partial}{\partial x_{i}}$ yields the identical relation between $e_{k-1}(A)$ 's.

\section{Proof of Theorem 2.5}

In this section we prove Theorem 2.5, our main geometric result. We first discuss some generalities on Chow motives, and then proceed with the proof.

In general $A^{\bullet}$ does not satisfy a Künneth formula, so we only have a possibly noninjective and non-surjective map $A^{\bullet}(X)_{\mathbb{Q}}^{\otimes m} \rightarrow A^{\bullet}\left(X^{m}\right)_{\mathbb{Q}}$. By using Chow motives we will see that certain correspondences provide enough of a substitute for the Künneth formula for our purposes.

We say that a correspondence on $X$ is an element of $A^{\bullet}\left(X^{2}\right)_{\mathbb{Q}}$. Let $\Gamma$ be a correspondence on $X$, which induces an endomorphism of $A^{\bullet}(X)_{\mathbb{Q}}$ via

$$
\Gamma: \alpha \mapsto\left(\pi_{2}\right)_{*}\left(\left(\pi_{1}^{*} \alpha\right) \cap \Gamma\right)
$$

where $\pi_{i}$ is the projection $X^{2} \rightarrow X$ onto the $i$ 'th factor. For any space $S, \Gamma$ similarly induces an endomorphism of $A^{\bullet}(S \times X)_{\mathbb{Q}}$. There is a notion of composition of correspondences, which for $\Gamma_{1}, \Gamma_{2} \in A^{\bullet}\left(X^{2}\right)_{\mathbb{Q}}$ is defined by

$$
\Gamma_{2} \circ \Gamma_{1}=\left(\pi_{13}\right)_{*}\left(\pi_{12}^{*} \Gamma_{1} \cup \pi_{23}^{*} \Gamma_{2}\right)
$$

where $\pi_{i j}$ is the projection $X^{3} \rightarrow X^{2}$ onto the $i, j$ factors. On the level of functions, $\Gamma_{2} \circ \Gamma_{1}$ induces the composite endomorphism of $A^{\bullet}(S \times X)_{\mathbb{Q}}$. Suppose that $\Gamma$ is 
idempotent, which means that

$$
\Gamma=\Gamma \circ \Gamma
$$

In particular, $\Gamma \in A^{\operatorname{dim}(X)}\left(X^{2}\right)_{\mathbb{Q}}$ so the associated function doesn't shift the grading. We remark that an effective Chow motive is a pair of the form $(X, \Gamma)$ with $\Gamma$ an idempotent correspondence on $X$. The identity for composition is the idempotent $\Gamma=\Delta_{2}$, whose associated endomorphism on $A^{\bullet}(S \times X)_{\mathbb{Q}}$ is the identity.

As $\Gamma$ is idempotent and $\Delta_{2}$ corresponds to the identity, $\Gamma, \Delta_{2}-\Gamma$ are orthogonal idempotents, so the images of their associated functions $A^{\bullet}(X)_{\mathbb{Q}} \rightarrow A^{\bullet}(X)_{\mathbb{Q}}$ direct sum to $A^{\bullet}(X)_{\mathbb{Q}}$. More generally, for $k \in\{m, n\}$, the $2^{k}$ elements $\Gamma^{\bigotimes\{1, \ldots, k\} \backslash B} \otimes$ $\left(\Delta_{2}-\Gamma\right)^{\otimes B}$ form an orthogonal system of idempotent correspondences of $X^{k}$ in $A^{\bullet}\left(\left(X^{2}\right)^{k}\right)_{\mathbb{Q}}=A^{\bullet}\left(\left(X^{k}\right)^{2}\right)_{\mathbb{Q}}$ summing to the identity correspondence $\Delta_{2}^{\bigotimes\{1, \ldots, k\}}$, so

$$
\begin{aligned}
A^{\bullet}\left(X^{m}\right)_{\mathbb{Q}} & =\bigoplus_{B \subset\{1, \ldots, m\}} \operatorname{Im}\left(\Gamma^{\bigotimes\{1, \ldots, m\} \backslash B} \otimes\left(\Delta_{2}-\Gamma\right)^{\bigotimes B}\right), \text { and } \\
A^{\bullet}\left(X^{n}\right)_{\mathbb{Q}} & =\bigoplus_{B \subset\{1, \ldots, n\}} \operatorname{Im}\left(\Gamma^{\bigotimes\{1, \ldots, n\} \backslash B} \otimes\left(\Delta_{2}-\Gamma\right)^{\bigotimes B}\right) .
\end{aligned}
$$

We remark that on any product space $X_{1} \times X_{2}$ with correspondences $\Gamma_{i} \in A^{\bullet}\left(\left(X_{i}\right)^{2}\right)_{\mathbb{Q}}$ we have $\Gamma_{1} \otimes \Gamma_{2}=\left(\Gamma_{1} \otimes\left(\Delta_{2}\right)_{X_{2}}\right) \circ\left(\left(\Delta_{2}\right)_{X_{1}} \otimes \Gamma_{2}\right)$. Hence for $k \in\{m, n\}$, each of the above correspondences is the composition of $k$ commuting correspondences on $X^{k}$, with the $i^{\prime}$ th correspondence inducing the endomorphism of $A^{\bullet}\left(X^{i-1} \times X \times X^{k-i}\right)_{\mathbb{Q}}$ from the associated correspondence on $X$.

It is clear that $\alpha \in A^{\bullet}\left(X^{m}\right)_{\mathbb{Q}}^{S_{m}}$ if and only if the component of $\alpha$ in $\operatorname{Im}\left(\Gamma^{\otimes\{1, \ldots, m\} \backslash B} \otimes\right.$ $\left.\left(\Delta_{2}-\Gamma\right)^{\otimes B}\right)$ is $S_{\{1, \ldots, m\} \backslash B} \times S_{B}$-invariant and depends only on $|B|$, and hence

$$
A^{\bullet}\left(X^{m}\right)_{\mathbb{Q}}^{S_{m}} \cong \bigoplus_{k=0}^{m} \operatorname{Im}\left(\Gamma^{\bigotimes m-k} \otimes\left(\Delta_{2}-\Gamma\right)^{\bigotimes k}\right)^{S_{m-k} \times S_{k}} .
$$

Note that Im does not distribute over $\nabla$ in general because there may be classes in $A^{\bullet}\left(X^{n}\right)_{\mathbb{Q}}$ which are not in the image of $A^{\bullet}(X)_{\mathbb{Q}}^{\otimes m} \rightarrow A^{\bullet}\left(X^{m}\right)_{\mathbb{Q}}$.

Proof of Theorem 2.5. Let $\Gamma=\gamma^{*} \otimes \gamma \in A^{\bullet}\left(X^{2}\right)_{\mathbb{Q}}$ with $\gamma^{*} \in A^{\bullet}(X)$. The idempotency of $\Gamma$ is equivalent to $\int \gamma \cup \gamma^{*}=1 \in A^{\bullet}(\mathrm{pt})_{\mathbb{Q}}$, and the map $\Gamma$ takes $\alpha \mapsto \gamma \cup \int\left(\alpha \cup \gamma^{*}\right)$. More generally, in any space $X \times Y, \Gamma$ induces the endomorphism of $A^{\bullet}(X \times Y)_{\mathbb{Q}}$

$$
\Gamma: \alpha \mapsto \gamma \otimes \int_{X \times Y \rightarrow Y} \alpha \cup\left(\gamma^{*} \otimes 1\right) .
$$

It directly follows that for this choice of $\Gamma$ we have

$$
\begin{aligned}
\operatorname{Im}\left(\Gamma^{\bigotimes m-k} \otimes\left(\Delta_{2}-\Gamma\right)^{\bigotimes k}\right)^{S_{m-k} \times S_{k}} & =\gamma^{\bigotimes m-k} \otimes \operatorname{Im}\left(\left(\Delta_{2}-\Gamma\right)^{\bigotimes k}\right)^{S_{k}} \\
\operatorname{Im}\left(\Gamma^{\bigotimes\{1, \ldots, n\} \backslash B} \otimes\left(\Delta_{2}-\Gamma\right)^{\bigotimes B}\right) & =\gamma^{\bigotimes\{1, \ldots, n\} \backslash B} \otimes \operatorname{Im}\left(\left(\Delta_{2}-\Gamma\right)^{\bigotimes B}\right) .
\end{aligned}
$$

Under the map $\alpha \mapsto \alpha(A)$, the $k^{\prime}$ th summand $\gamma^{\bigotimes m-k} \otimes \operatorname{Im}\left(\left(\Delta_{2}-\Gamma\right)^{\bigotimes k}\right)^{S_{m-k} \times S_{k}}$ of $A^{\bullet}\left(X^{m}\right)^{S_{m}}$ maps to

$$
\bigoplus_{|B|=k} \gamma^{\bigotimes\{1, \ldots, n\} \backslash B} \otimes \operatorname{Im}\left(\left(\Delta_{2}-\Gamma\right)^{\bigotimes B}\right) \subset A^{\bullet}\left(X^{n}\right)_{\mathbb{Q}},
$$


which are disjoint summands for distinct $k$ 's. This map acts on an element $\gamma^{\otimes k} \otimes \beta \in$ $\gamma^{\bigotimes m-k} \otimes \operatorname{Im}\left(\left(\Delta_{2}-\Gamma\right)^{\bigotimes k}\right)^{S_{m-k} \times S_{k}}$ in the $k^{\prime}$ th summand of $A^{\bullet}\left(X^{m}\right)^{S_{m}}$ via

$$
\gamma^{\bigotimes m-k} \otimes \beta \mapsto \sum_{|B|=k, B \subset A} \gamma^{\bigotimes\{1, \ldots, n\} \backslash B} \otimes \beta .
$$

Note that there is exactly one term in each summand $\gamma^{\bigotimes\{1, \ldots, n\} \backslash B} \otimes \operatorname{Im}((\Delta-$ $\left.\Gamma)^{\bigotimes B}\right) \subset A^{\bullet}\left(X^{n}\right)_{\mathbb{Q}}$ with $|B|=k$ and $B \subset A$, and the term depends only on $\beta$ and not on $A$.

We may check directly that

$$
\left(\Gamma^{\bigotimes\{1, \ldots, m\} \backslash B} \otimes(\Delta(2)-\Gamma)^{\bigotimes B}\right)(\alpha)=\gamma^{\{1, \ldots, m\} \backslash B} \otimes \alpha_{|B|}^{\prime},
$$

so the component of $\alpha$ in the $k$ 'th summand $\operatorname{Im}\left(\Gamma^{\bigotimes m-k} \otimes\left(\Delta_{2}-\Gamma\right)^{\bigotimes k}\right)^{S_{m-k} \times S_{k}}$ is $\gamma^{m-k} \otimes \alpha_{k}^{\prime}$.

Hence, if we set

$$
f=e_{k_{1}}+\ldots+e_{k_{r}} \in \mathbb{Z}\left[x_{1}, \ldots, x_{m}\right]^{S_{m}}
$$

where the $k_{i}$ are those $k$ such that $\gamma^{\{1, \ldots, n\} \backslash B} \otimes \alpha_{k}^{\prime} \neq 0$ for any $|B|=k$ (note that by symmetry either the classes for all such $B$ vanish or none vanish), then the $\mathbb{Z}$-linear relations between the $f(A)$ polynomials are identical to the $\mathbb{Z}$-linear relations between the $\alpha(A)$ for $A$ ranging over $m$-element subsets of $\{1, \ldots, n\}$.

Finally,

$$
\operatorname{Im}\left((\Delta-\Gamma)^{\bigotimes B}\right) \cong \gamma^{\bigotimes\{1, \ldots, n\} \backslash B} \otimes \operatorname{Im}\left((\Delta-\Gamma)^{\bigotimes B}\right)
$$

because the map $\beta \mapsto \gamma^{\bigotimes\{1, \ldots, n\} \backslash B} \otimes \beta$ has an inverse map given by $\delta \mapsto \int_{X^{n} \rightarrow X^{B}} \delta \cup$ $\left(\gamma^{*}\right)^{\bigotimes\{1, \ldots, n\} \backslash B}$, so we have

$$
\gamma^{\bigotimes\{1, \ldots, n\} \backslash B} \otimes \alpha_{|B|}^{\prime}=0 \Longleftrightarrow \alpha_{|B|}^{\prime}=0 .
$$

The result now follows by applying Corollary 2.4 to $f$.

Remark 4.1. We remark that there is a notion of homomorphism and tensor product for Chow motives, and if $\gamma \in A^{k}(X)_{\mathbb{Q}}$, then $\left(X, \gamma^{*} \otimes \gamma\right)$ is isomorphic to the motive $\mathbb{L}^{\otimes k}$ where $\mathbb{L}$ is the Lefschetz motive $\left(\mathbb{P}^{1}, \mathbb{P}^{1} \times\{\mathrm{pt}\}\right)$, see [Kim05].

Acknowledgements. I would like to thank Dennis Tseng for suggesting to study relations between diagonals and for many fruitful conversations.

\section{REFERENCES}

[BV04] Arnaud Beauville and Claire Voisin. On the Chow ring of a $K 3$ surface. J. Algebraic Geom., 13(3):417-426, 2004.

[GS95] B. H. Gross and C. Schoen. The modified diagonal cycle on the triple product of a pointed curve. Ann. Inst. Fourier (Grenoble), 45(3):649-679, 1995.

[Kim05] Shun-Ichi Kimura. Chow groups are finite dimensional, in some sense. Math. Ann., 331(1):173-201, 2005.

[MY16] Ben Moonen and Qizheng Yin. Some remarks on modified diagonals. Commun. Contemp. Math., 18(1):1550009, 16, 2016.

[O'G14] Kieran G. O'Grady. Computations with modified diagonals. Atti Accad. Naz. Lincei Rend. Lincei Mat. Appl., 25(3):249-274, 2014.

[Qiz14] Yin Qizheng. Tautological cycles on curves and jacobians. thesis. radboud university nijmegen. 2014.

[Voi15] Claire Voisin. Some new results on modified diagonals. Geom. Topol., 19(6):3307-3343, 2015. 